\title{
Quality of Life in Patients with Metastatic Gastroenteropancreatic Neuroendocrine Tumors Receiving Peptide Receptor Radionuclide Therapy: Information from a Monitoring Program in Clinical Routine
}

\author{
Caroline Martini ${ }^{1}$, Sabine Buxbaum ${ }^{2}$, Margarida Rodrigues ${ }^{2}$, Bernhard Nilica ${ }^{2}$, Lorenza Scarpa ${ }^{2}$, Bernhard Holzner ${ }^{1,3}$, \\ Irene Virgolini ${ }^{2}$, and Eva-Maria Gamper ${ }^{4}$ \\ ${ }^{1}$ Department of Psychiatry, Psychotherapy, and Psychosomatics, Psychiatry I, Medical University of Innsbruck, Innsbruck, Austria; \\ ${ }^{2}$ Department of Nuclear Medicine, Medical University of Innsbruck, Innsbruck, Austria; ${ }^{3}$ Department of Psychiatry, Psychotherapy \\ and Psychosomatics, Psychiatry II, Medical University of Innsbruck, Innsbruck, Austria; and ${ }^{4}$ Innsbruck Institute of Patient-Centered \\ Outcome Research (IIPCOR), Innsbruck, Austria
}

In patients with metastatic gastroenteropancreatic neuroendocrine tumors (NETs), we evaluated health-related quality of life (HRQoL) from the first peptide receptor radionuclide therapy (PRRT) to the first restaging and compared the scores with general-population (GP) norms. Methods: The data were from routine HRQoL monitoring using the core quality-of-life questionnaire of the European Organization for Research and Treatment of Cancer (EORTC QLQ-C30). Patients received 4-6 cycles of ${ }^{177} \mathrm{Lu}$-DOTATATE or ${ }^{90} \mathrm{Y}-$ DOTATOC. To be eligible for analysis, patients had to have at least one HRQoL assessment before PRRT and at least one HRQoL assessment at the end of or after treatment completion. Linear mixed models were used to consider HRQoL changes over time. Results: In total, 61 gastroenteropancreatic NET patients (small-intestine NETs, $n=37$; pancreatic NETs, $n=24$ ) were eligible for analysis. Clear improvements from baseline to the first restaging were found for diarrhea in small-intestine NET patients, showing a decrease of 16 points, which represents a moderately large change. We observed a clinically relevant decrease in appetite loss (17 points), but for female small-intestine NET patients only. Other HRQoL changes were also restricted to sociodemographic or clinical subgroups and mainly reflected improvements, except for physical and social functioning, which showed decreasing scores in older small-intestine NET patients. Compared with HRQoL GP norms, patients had impairments consisting of diarrhea; fatigue; appetite loss; reduced physical, social, and role functioning; and reduced global HRQoL. Except for diarrhea and appetite loss, patient scores at the first restaging did not reach GP levels. Conclusion: Our analyses support previous findings of overall stable HRQoL under PRRT. Yet, significant HRQoL impairments compared with the GP and potentially specific subgroup patterns need to be considered.

\footnotetext{
Received Dec. 14, 2017; revision accepted Jan. 31, 2018.

For correspondence or reprints contact: Eva-Maria Gamper, Innsbruck Institute of Patient-Centered Outcome Research (IIPCOR), Dr.-Stumpf-Straße 56, 6020 Innsbruck, Austria.

E-mail: eva.gamper@iipcor.org

Published online Jul. 24, 2018.

COPYRIGHT (C 2018 by the Society of Nuclear Medicine and Molecular Imaging.
}

Key Words: gastroenteropancreatic neuroendocrine tumors; peptide receptor radionuclide therapy; health-related quality of life; clinical practice; routine monitoring

J Nucl Med 2018; 59:1566-1573

DOI: 10.2967/jnumed.117.204834

$\mathbf{G}_{\mathbf{a}}$ astroenteropancreatic neuroendocrine tumors (GEPNETs) are considered a relatively rare disease, although incidence rates have almost doubled over the past 3 decades $(1,2)$. They may be asymptomatic for years and are often diagnosed at an advanced stage (3-5). Progress in the management of the disease has contributed to increased long-term survival rates $(2,6,7)$ and therefore to an increasing percentage of patients in palliative care, where health-related quality of life (HRQoL) is of special significance $(3,8)$.

Although HRQoL is an important outcome in clinical trials in oncology, its assessment in the field of neuroendocrine tumor (NET) research has a young tradition, and knowledge is still limited $(9,10)$. Evidence from studies including heterogeneous clinical subgroups suggests that NET patients perceive their overall HRQoL as relatively good (11-13). However, specific physical and psychosocial complaints are often reported, such as poor physical, emotional, and social functioning; impaired sleep; and significant levels of fatigue (10-17). Molecularly targeted treatments, such as peptide receptor radionuclide therapy (PRRT), have been shown effective in terms of both symptomatic control and survival $(8,18-21)$. PRRT is generally well tolerated (18,21-23), and evidence from studies including patient outcomes suggests favorable outcomes in terms of HRQoL (20,24-29).

In recent years, there has been growing awareness of the need to incorporate HRQoL assessment not only into clinical trials but also into routine clinical practice, where it has a positive impact on a range of patient and clinical care outcomes (30-33). Such real-world data from outside an idealized study setting add valuable information on patients' perceptions of disease and treatment to those obtained from randomized controlled trials (34).

To the best of our knowledge, no publication has yet reported routine HRQoL data obtained from NET patients under the real-world conditions of daily clinical routine. Therefore, we aimed to analyze such data in metastatic GEPNET patients receiving a first PRRT. 
TABLE 1

Sociodemographic and Clinical Characteristics for Small-Intestine and Pancreatic NET Patients

\begin{tabular}{|c|c|c|}
\hline Characteristic & Small-intestine NETs $(n=37)$ & Pancreatic NETs $(n=24)$ \\
\hline \multicolumn{3}{|l|}{ Age (y) } \\
\hline Mean & $62.8(\mathrm{SD}, 11.9)$ & $61.0(\mathrm{SD}, 12.6)$ \\
\hline Range & $37-88$ & $37-88$ \\
\hline \multicolumn{3}{|l|}{$\operatorname{Sex}(n)$} \\
\hline Male & $22(59.5 \%)$ & $15(62.5 \%)$ \\
\hline Female & $15(40.5 \%)$ & $9(37.5 \%)$ \\
\hline \multicolumn{3}{|l|}{ Marital status $(n)$} \\
\hline Single & $4(10.8 \%)$ & $3(12.5 \%)$ \\
\hline Partnership/marriage & $24(64.9 \%)$ & $18(75.0 \%)$ \\
\hline Divorced/separated & $5(13.5 \%)$ & $1(4.2 \%)$ \\
\hline Widowed & $3(8.1 \%)$ & $2(8.3 \%)$ \\
\hline Missing & $1(2.7 \%)$ & - \\
\hline \multicolumn{3}{|l|}{ Employment status $(n)$} \\
\hline Employed & $4(10.8 \%)$ & $3(12.5 \%)$ \\
\hline Self-employed & $1(2.7 \%)$ & $20(83.3 \%)$ \\
\hline Retired & $28(75.7 \%)$ & $1(4.2 \%)$ \\
\hline Missing & $4(10.8 \%)$ & - \\
\hline \multicolumn{3}{|l|}{ Karnofsky score at baseline } \\
\hline Mean & $98.5(\mathrm{SD}, 3.6)$ & $91.4(\mathrm{SD}, 12.0)$ \\
\hline Range & $90-100$ & $60-100$ \\
\hline \multicolumn{3}{|l|}{$\operatorname{PRRT}(n)$} \\
\hline${ }^{177} \mathrm{Lu}$ & $26(70.3 \%)$ & $15(62.5 \%)$ \\
\hline${ }^{90} Y$ & $11(29.7 \%)$ & 9 (37.5\%) \\
\hline \multicolumn{3}{|l|}{ PRRT cycles received $(n)$} \\
\hline 3 cycles & - & $2(8.3 \%)$ \\
\hline 4 cycles & $29(85.3 \%)$ & $19(79.2 \%)$ \\
\hline 5 cycles & $4(10.8 \%)$ & $3(12.5 \%)$ \\
\hline 6 cycles & $1(2.7 \%)$ & - \\
\hline \multicolumn{3}{|l|}{ Previous treatment $(n)$} \\
\hline Surgery & $25(67.6 \%)$ & 8 (33.3\%) \\
\hline Chemotherapy & $5(13.5 \%)$ & $6(25.0 \%)$ \\
\hline Radiation therapy & $1(2.7 \%)$ & $1(4.2 \%)$ \\
\hline Biologic therapy & $3(8.1 \%)$ & $2(8.3 \%)$ \\
\hline Targeted therapy & $3(8.1 \%)$ & $2(8.3 \%)$ \\
\hline Chemoembolization & $1(2.7 \%)$ & - \\
\hline Radiofrequency ablation & $2(5.4 \%)$ & - \\
\hline Somatostatin analogs & 32 (86.5\%) & $12(50.0 \%)$ \\
\hline \multicolumn{3}{|l|}{ HRQoL assessments $(n)$} \\
\hline $\mathrm{T} 1$ & 37 (100.0\%) & $24(100.0 \%)$ \\
\hline T2 & $28(75.7 \%)$ & 19 (79.2\%) \\
\hline T3 & $27(73.0 \%)$ & $17(70.8 \%)$ \\
\hline $\mathrm{T} 4$ & $26(70.3 \%)$ & $14(58.3 \%)$ \\
\hline \multicolumn{3}{|c|}{ Progression within 1 y after first PRRT $(n)$} \\
\hline Yes & $10(27.0 \%)$ & $8(33.3 \%)$ \\
\hline No & $21(56.8 \%)$ & $15(62.5 \%)$ \\
\hline Missing & $6(16.2 \%)$ & $1(4.2 \%)$ \\
\hline
\end{tabular}


Our first aim was to investigate HRQoL of metastatic GEPNET patients over the course of first PRRT. Two hypotheses were tested: the first was that small-intestine and pancreatic NET patients would show changes in fatigue, pain, nausea/vomiting, appetite loss, physical, social, role, and emotional functioning between baseline and first restaging. The second was that small intestine NET patients additionally would show changes in diarrhea from baseline to first restaging. Changes in other HRQoL aspects were investigated on an explorative basis.

The second study aim was to compare patients' HRQoL scores with those of a matched sample from the Austrian general population (GP).

\section{MATERIALS AND METHODS}

\section{Patients and Data Collection}

The dataset used for analyses was obtained from HRQoL monitoring at the Department of Nuclear Medicine, Medical University of Innsbruck. Patients are admitted to the department for treatment or follow-up examinations involving radiopharmaceuticals. The routine course at our site is staging with ${ }^{68} \mathrm{Ga}$-DOTATOC PET, 4 cycles of PRRT every $10-12 \mathrm{wk}$, and restaging after 3 mo with ${ }^{68} \mathrm{Ga}$-DOTATOC PET and at 6-mo intervals thereafter. The radionuclides used are ${ }^{90} \mathrm{Y}-$ DOTATOC $\left({ }^{90} \mathrm{Y}\right)$, ideally for single, larger lesions, and ${ }^{177} \mathrm{Lu}$-DOTATATE $\left({ }^{177} \mathrm{Lu}\right)$, for smaller lesions. However, periods of nonavailability did not allow for a consistent following of this strategy.

Patients were invited to participate in HRQoL monitoring at each inpatient visit. The eligibility criteria for HRQoL monitoring were a diagnosis of cancer, an age of at least $18 \mathrm{y}$, no brain metastases, and no diagnosis of dementia or overt cognitive impairment. Patients were approached by the nursing staff during the admission procedure and asked to complete the core quality-of-life questionnaire (QLQ-C30) of the European
Organization for Research and Treatment of Cancer (EORTC). More details on the monitoring have been provided by Gamper et al. (35).

We obtained HRQoL information from the monitoring dataset, and we obtained sociodemographic and clinical data from medical records. For this type of retrospective investigation, no separate ethical approval and no formal consent are required by Austrian law.

Age- and sex-matched population-based controls were taken from a set of previously collected normative data for the EORTC QLQ-C30, including 2,000 subjects of the Austrian GP. Details on sampling and data collection are provided elsewhere (36).

\section{HRQoL Assessment Time Points}

From the large number of HRQoL assessments, we extracted those related to 4 clinically relevant time points: T1, the time of admission for the first PRRT cycle (before administration, with no octreotide 4 wk beforehand); T2, the time of admission for the second PRRT cycle ( 2 mo after baseline); T3, the time of admission for the last PRRT cycle (cycles 4-6; 4-6 mo after baseline); and T4, the time of admission for the first restaging ( $3 \mathrm{mo}$ after the last cycle, with no octreotide $4 \mathrm{wk}$ beforehand). To be included in the analysis, patients had to have completed at least a T1 and a T3 or T4 HRQoL assessment.

\section{HRQoL Questionnaire}

HRQoL was assessed using the EORTC QLQ-C30 (37), one of the most widely used cancer-specific HRQoL questionnaires with good psychometric properties. It consists of 30 items constituting scales of 5 functions (physical, role, emotional, social, and cognitive), 9 symptoms and single items (fatigue, nausea/vomiting, pain, dyspnea, insomnia, appetite loss, constipation, diarrhea, and financial difficulties), and global HRQoL. Raw scores were linearly transformed to a scale of $0-100$, with
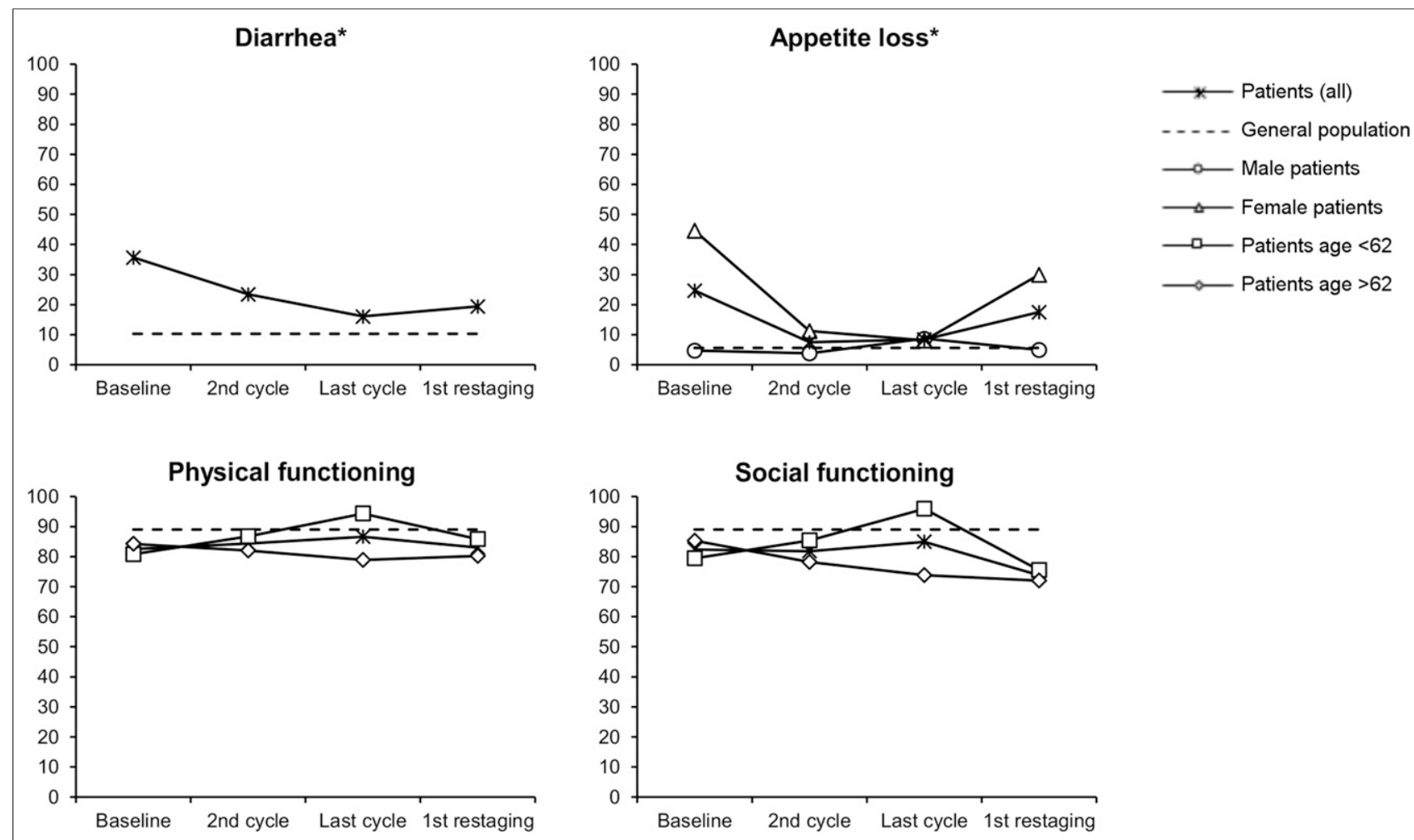

*no statistically significant difference between mean scores at $1^{\text {st }}$ restaging and GP scores

FIGURE 1. Course of HRQoL domains with significant changes between baseline and first restaging in small-intestine NET patients compared with GP. 
higher scores reflecting a higher level of functioning and symptomatology, respectively (38). A change of 5-10 points in EORTC QLQ-C30 mean scores was considered small, whereas a change of 10-20 points was considered moderate and a change of more than 20 points as large (39).

\section{Statistical Analysis}

Statistical analyses were performed using IBM SPSS, version 22.0.

The course of HRQoL from T1 to T4 was analyzed separately for small-intestine and pancreatic NETs, as they were considered to be associated with different HRQoL issues. Linear mixed models were used to handle unbalanced data. To account for correlations between repeated assessments, we used a first-order autoregressive covariance structure and included subject as a random factor. The EORTC QLQC30 domains were included as dependent variables. Assessment time point (T1-T4), sex, age (dichotomized at median), progression within $1 \mathrm{y}$ after treatment completion (yes/no), and radionuclide for PRRT $\left({ }^{90} \mathrm{Y} /{ }^{177} \mathrm{Lu}\right)$ were included as independent variables. Interaction effects between assessment time points and other independent variables were tested to investigate whether the course of HRQoL differed between subgroups. We used an $\alpha$-level of 0.5 or less for hypothesis testing and of 0.1 or less for explorative analyses.

Two-way ANOVA was performed to determine HRQoL differences between GEPNET patients and the GP, with group (patients/GP), sex, and age as factors.

\section{RESULTS}

\section{Patient Characteristics}

From 2005 to 2014, 133 GEPNET patients were eligible for routine HRQoL monitoring. Of these, 37 small-intestine NET patients (mean age, $62.8 \mathrm{y} ; 40.5 \%$ female) and 24 pancreatic NET patients (mean age, $61.0 \mathrm{y} ; 37.5 \%$ female) completed at least a T1
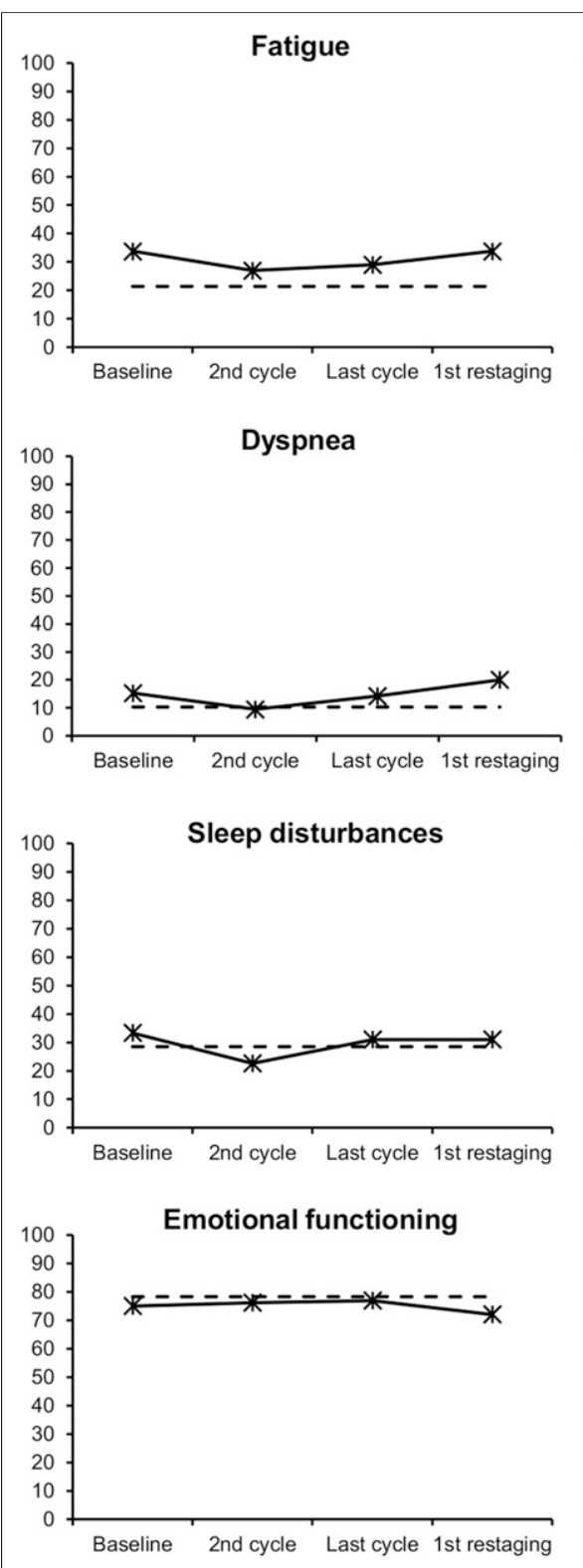
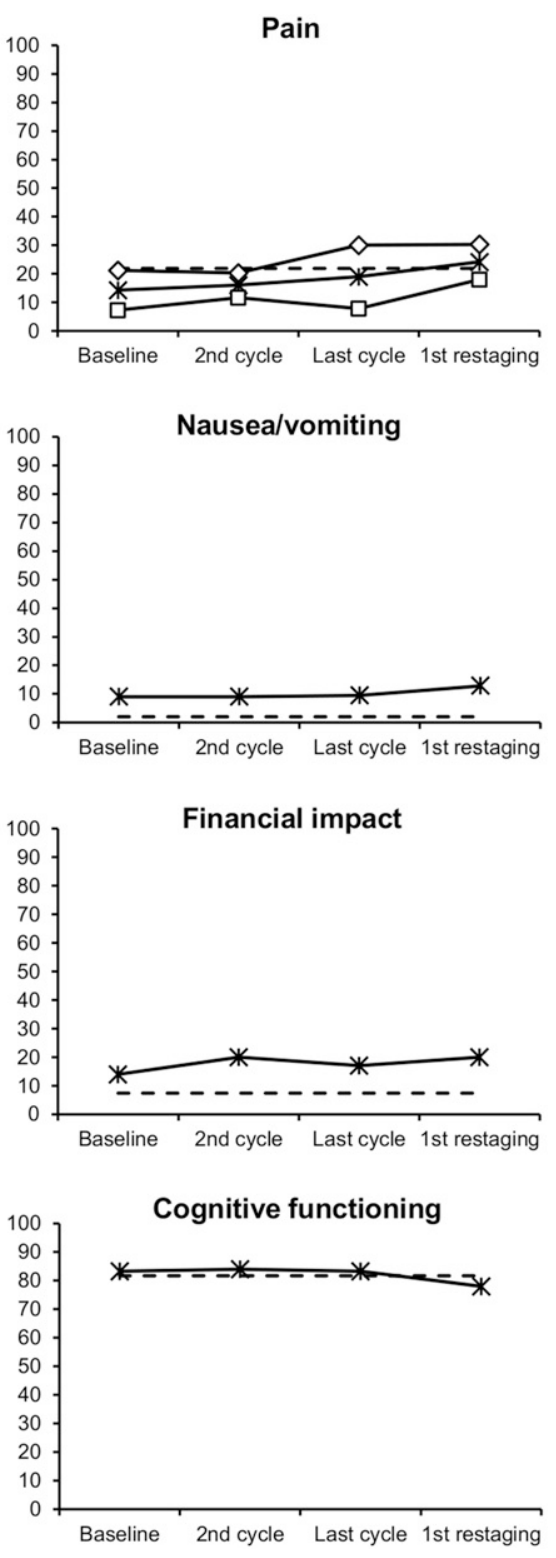
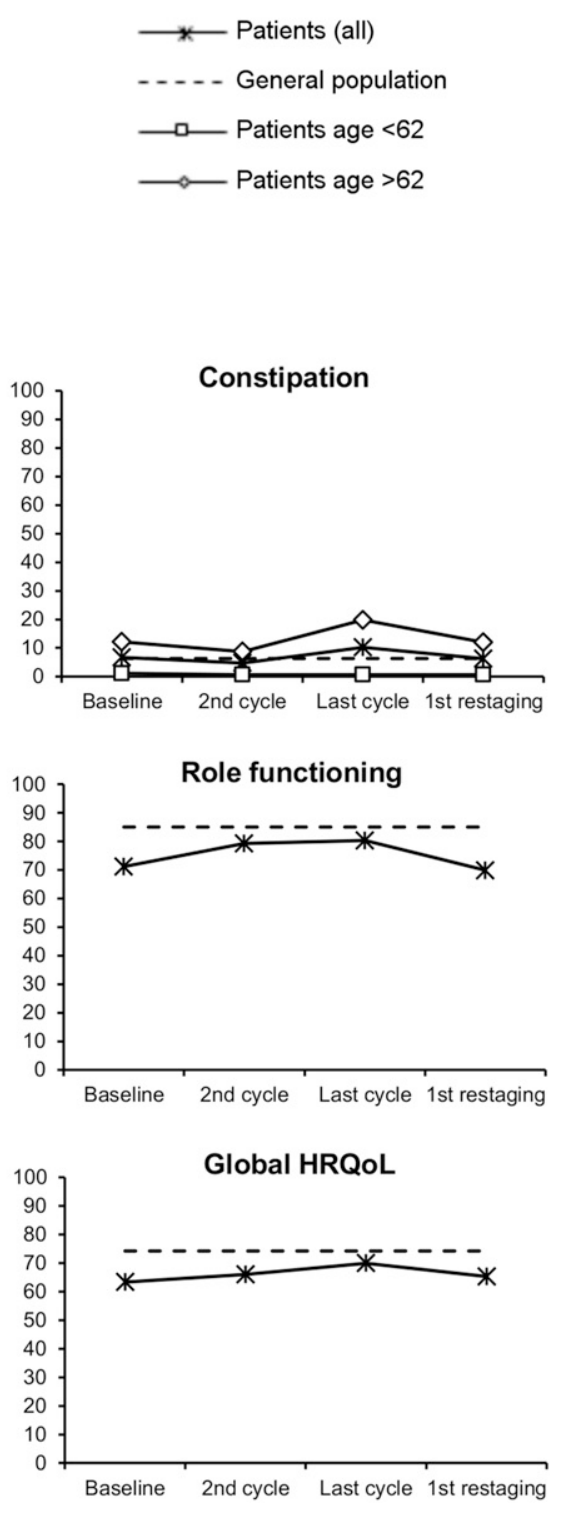

FIGURE 2. Course of HRQoL domains without significant changes between baseline and first restaging in small-intestine NET patients compared with GP. 
and a T3 or T4 HRQoL assessment and were therefore eligible for analysis. PRRT was given as the first-line treatment to 30 small-intestine NET patients $(81.1 \%)$ and 17 pancreatic NET patients $(70.8 \%) .{ }^{90} \mathrm{Y}$ was used in $29.7 \%$ of small-intestine and $37.5 \%$ of pancreatic NET patients. Most patients completed 4 cycles of PRRT. Details on sociodemographic and clinical characteristics are provided in Table 1.

\section{HRQoL in Small-Intestine NET Patients}

Comparison of small-intestine NET patients' HRQoL scores at T1 with those of GP controls revealed clinically relevant differences to the detriment of patients for diarrhea $(+26.6$ points, $P<0.001)$, appetite loss $(+15.9$ points, $P=0.009)$, fatigue $(+13.2$ points, $P=0.024)$, physical functioning $(-10.0$ points, $P=0.036)$, social functioning $(-13.1$ points, $P=0.014)$, role functioning $(-14.8$ points, $P=$ 0.033), and global HRQoL ( -11.6 points, $P=0.019)$.

In the course of HRQoL from T1 to T4, the most pronounced change in small-intestine NET patients was a clinically relevant improvement in diarrhea $(-16.3$ points, $P=0.008)$. An improvement over time was also found for appetite loss, but in women only (interaction, $P=0.001$ ). Female patients had relatively high baseline scores, which, despite a significant and clinically relevant decrease of 17 points, were still clearly higher $(+22$ points $)$ at $\mathrm{T} 4$ than male patients' scores. For the functioning domains, change patterns were less clear. Significant interaction terms between assessment time point and age for physical functioning $(P=0.044)$ and social functioning $(P=0.035)$ indicated age-related effects. Baseline functioning levels for both domains were higher in patients above the median age of $62 \mathrm{y}$ than in those $62 \mathrm{y}$ or younger, and the course of functioning from $\mathrm{T} 1$ to $\mathrm{T} 4$ differed between age groups, with older patients reporting decreasing scores. The mean decrease in social functioning, shown in Figure 1, was not statistically significant after accounting for the interaction with age. No changes from T1 to T4 were found in any of the other domains. Overall, patients older than 62 y reported significantly more pain than younger patients. Mean score differences between T1 and T4, shown in Figure 2, did not reach statistical significance.

At T4, mean differences regarding fatigue and the functioning domains were smaller but still statistically significant (fatigue, +3.7 points, $P=0.017$; physical functioning, -3.1 points, $P=$ 0.013; social functioning, -5.7 points, $P=0.001$; role functioning, -4.2 points, $P=0.019$ ) (not accounting for age group differences), whereas for diarrhea and appetite loss (not accounting for sex differences) there was no longer a statistically significant difference from GP scores (diarrhea, +2.8 points, $P=0.070$; appetite loss, +3.2 points, $P=0.052$ ).

Figure 1 shows symptom and functioning trajectories over treatment, including GP scores for domains with significant changes between $\mathrm{T} 1$ and T4; the remaining domains are shown in Figure 2.

\section{HRQoL in Pancreatic NET Patients}

Significant interactions regarding social and emotional functioning indicated significant changes over time for specific treatment and sociodemographic subgroups only. Social functioning improved from $\mathrm{T} 1$ to $\mathrm{T} 4$ in patients treated with ${ }^{90} \mathrm{Y}(+37.1$ points). Scores approximated those of patients treated with ${ }^{177} \mathrm{Lu}$, which did not change significantly over time (interaction, $P=0.008$ ). Emotional functioning clearly improved in male patients ( +17.1 points), whereas it slightly decreased in female patients $(-5.7$ points) (interaction, $P=0.013$ ). For role functioning, we found no clear pattern for the significant interaction term $(P=0.005)$. No changes from $\mathrm{T} 1$ to $\mathrm{T} 4$ were found in any of the other domains.
Differences on a range of domains in association with the type of radionuclide were found. Compared with ${ }^{90} \mathrm{Y},{ }^{177} \mathrm{Lu}$ was associated with less fatigue $(-27.7$ points, $P=0.020)$ and better physical functioning $(+22.4$ points, $P=0.050)$, cognitive functioning $(+23.1$ points, $P=0.003)$, and global HRQoL $(+17.3$ points, $P=0.029$ ).

Statistical analyses to compare pancreatic NET patients' and GP's HRQoL scores were not performed, because of the limited number of patients. Figure 3 shows HRQoL trajectories over time for domains with significant changes; the remaining domains are shown in Figure 4.

\section{DISCUSSION}

The present investigation examined the course of HRQoL in patients with metastatic GEPNETs undergoing a first PRRT. We expected HRQoL changes over the course of treatment both as a result of efficient palliation of symptoms $(18,40,41)$ and as a result of accumulation of radioactivity toward the end of PRRT (42).

In small-intestine NET patients, we found significant impairments at baseline compared with the GP regarding physical, social, and role functioning; fatigue; diarrhea; and appetite loss, which have also been reported in NET patients receiving treatment other than PRRT $(14,16,17,43,44)$. Most of these differences were still observed at T4, except for diarrhea, a cardinal symptom in these patients, and appetite loss, both of which reached GP levels. Clinical studies on PRRT for GEPNETs showed HRQoL improvements in different symptom and functioning domains over the course of treatment $(20,24-29)$. In our analysis of small-intestine NET patients, most changes between $\mathrm{T} 1$ and $\mathrm{T} 4$ were observed in sociodemographic subgroups only. For appetite loss, we found women to report more symptoms than men throughout treatment and followup, with considerable improvements after baseline. Sex differences in the somatic experience of emotional distress (45-47) may be considered here. Besides the reported improvements, we found most

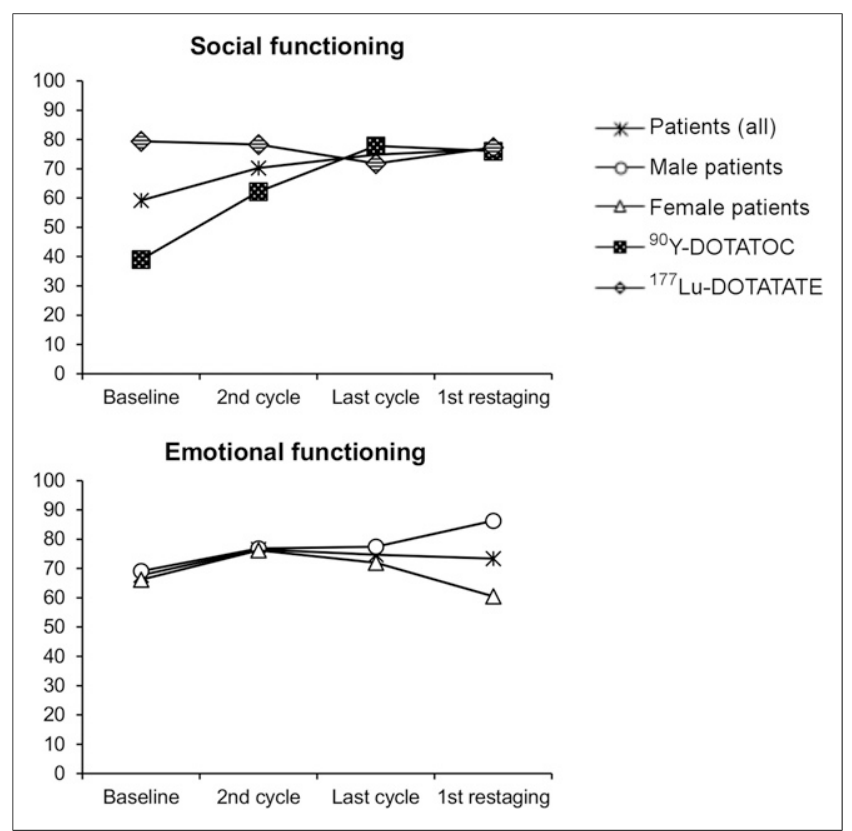

FIGURE 3. Course of HRQoL domains with significant changes between baseline and first restaging in pancreatic NET patients compared with GP. 


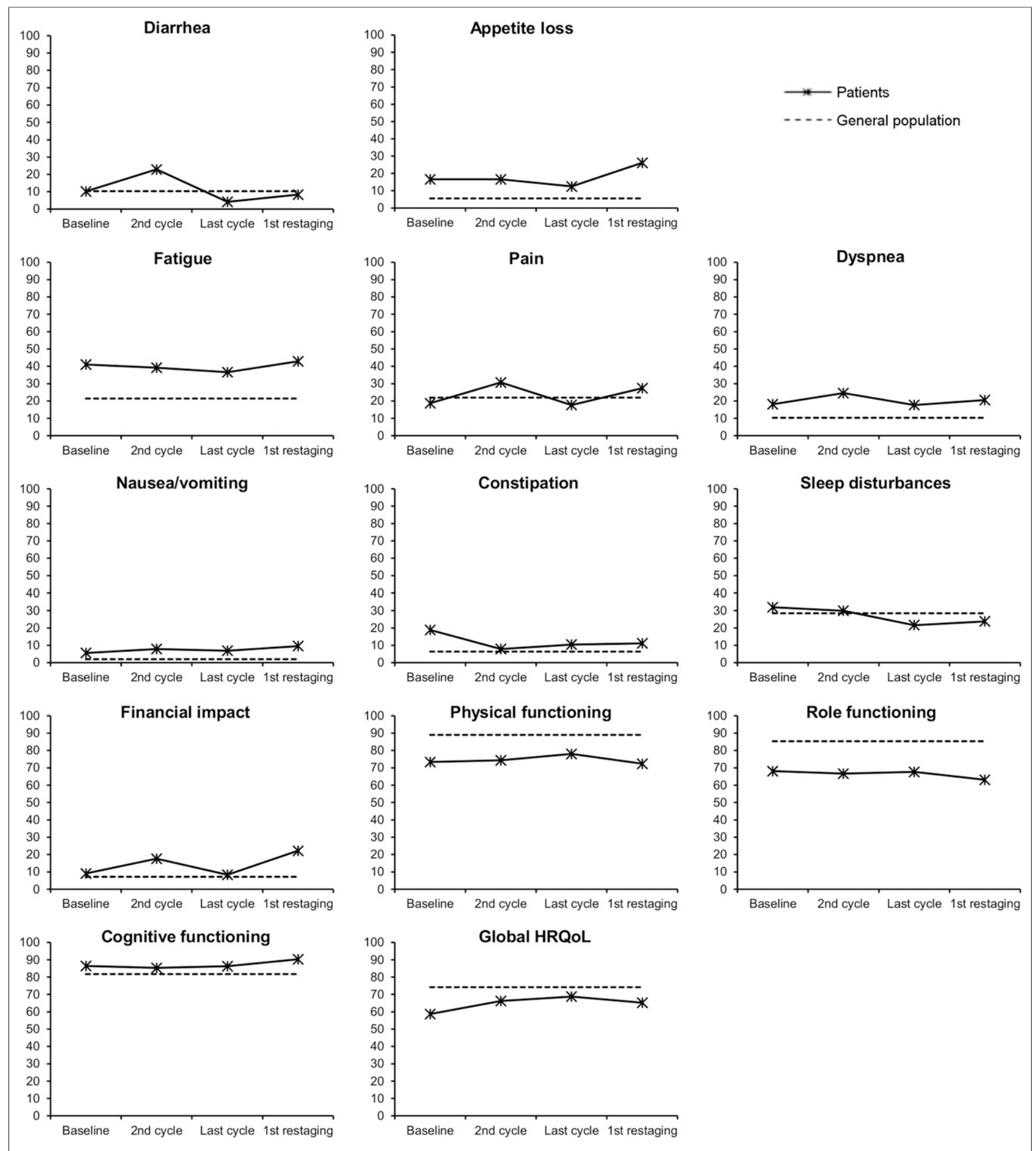

FIGURE 4. Course of HRQoL domains without significant changes between baseline and first restaging in pancreatic NET patients compared with GP.

HRQoL scores to be stable over time, except the worsening of physical and social functioning observed in patients older than $62 \mathrm{y}$, a finding that warrants further investigation of NET-specific issues not assessed by the EORTC QLQ-C30.

Interpretation of results from HRQoL assessments in pancreatic NET patients requires caution because of the small sample size. The main result here was that patients treated with ${ }^{90} \mathrm{Y}$ already had a lower HRQoL at baseline over a range of domains (e.g., physical functioning and global HRQoL) than those treated with ${ }^{177} \mathrm{Lu}$. These effects may be due to the fact that ${ }^{90} \mathrm{Y}$ usually is administered in patients with larger lesions, which may be associated with higher symptomatology. Unfortunately, we were not able to compare pancreatic NET patients' HRQoL scores with the GP, because of lack of statistical power. 
Placement of our results into the context of clinical GEPNET studies on targeted treatments, such as everolimus and sunitinib, is limited by the lack of HRQoL data in these patients (48). However, the available studies suggest that HRQoL is maintained over the course of treatment. In the phase 3 RADIANT-4 trial (49), where HRQoL was assessed as a secondary outcome, patients with advanced gastrointestinal or lung NETs treated with everolimus reported stable HRQoL over time, with no significant differences from a placebo group. Similarly, in a phase 3b study (50) HRQoL in patients with pancreatic NETs remained stable, whereas there was a slight decrease in patients with midgut NETs. Results from a phase 3 study of sunitinib (51) showed stable HRQoL, except for diarrhea and insomnia, which worsened with sunitinib compared with placebo. Largely consistent with these findings, we mainly observed stable or even improved HRQoL scores over the course of PRRT until the first restaging, further supporting existing evidence.

A major limitation of the present work was the small sample size. Because data are primarily collected for use in clinical routine, there are various reasons for missing questionnaires (e.g., patient admission timing). We performed a crude comparison of sociodemographic basic characteristics of the included and excluded patients and found no statistically significant differences regarding age or sex. However, we cannot exclude a selection bias, especially in terms of overrepresentation of patients with high baseline function according to the Karnofsky performance status, which was between 90 and 100 in most patients included in analysis. Also, because of the lack of a control group, we cannot make assumptions about the HRQoL-"preserving" effect of PRRT.

Another limitation was that we could draw information from only the core questionnaire, EORTC QLQ-C30, which covers general HRQoL aspects but may miss NET-specific issues. Currently, 2 NET-specific HRQoL instruments are available: the QLQ-GI.NET21 (52), a module to be applied together with EORTC QLQ-C30, and the Norfolk QOL-NET (53). Both of these NET-specific instruments include questions on, for example, endocrine and gastrointestinal symptoms, sexual functioning, and depression. In the light of emerging precision medicine concepts, such disease- or treatment-specific data may add considerable value to existing knowledge of HRQoL in these patients $(54,55)$. At our department, the QLQ-GI.NET21 has been implemented but has been administered to only a few patients so far.

Despite these limitations, a major strength of the present investigation is the perspective it provides on patients' HRQoL from outside a clinical study setting. Routinely collected HRQoL data are of great importance because they reflect a real-world pattern of treatment and care. Especially in rare diseases such as NETs, for which clinical studies with sufficient power are more difficult to conduct, such information can contribute to a better understanding of HRQoL issues. With the increasing efforts to integrate the patient's voice into the assessment of care quality, the use of HRQoL in performance measurements can contribute to the effectiveness with which treatments such as PRRT are evaluated $(56,57)$.

\section{CONCLUSION}

The present analysis of routine HRQoL data from patients with metastatic GEPNETs undergoing a first PRRT indicated improved or at least stable HRQoL on several domains from baseline to the first restaging. Although these results thereby support previous evidence from clinical PRRT studies on such patients, they also clearly show that patients have significant HRQoL impairments compared with the GP.

\section{DISCLOSURE}

The work of Caroline Martini was funded by grant P269300 from the Austrian Science Fund (FWF). No other potential conflict of interest relevant to this article was reported.

\section{REFERENCES}

1. Hallet J, Law CH, Cukier M, Saskin R, Liu N, Singh S. Exploring the rising incidence of neuroendocrine tumors: a population-based analysis of epidemiology, metastatic presentation, and outcomes. Cancer. 2015;121:589-597.

2. Yao JC, Hassan M, Phan A, et al. One hundred years after "carcinoid": epidemiology of and prognostic factors for neuroendocrine tumors in 35,825 cases in the United States. J Clin Oncol. 2008;26:3063-3072.

3. Ramage JK, Ahmed A, Ardill J, et al. Guidelines for the management of gastroenteropancreatic neuroendocrine (including carcinoid) tumours (NETs). Gut. 2012;61:6-32.

4. Chau I, Casciano R, Willet J, Wang X, Yao J. Quality of life, resource utilisation and health economics assessment in advanced neuroendocrine tumours: a systematic review. Eur J Cancer Care (Engl). 2013;22:714-725.

5. Frilling A, Sotiropoulos GC, Li J, Kornasiewicz O, Plöckinger U. Multimodal management of neuroendocrine liver metastases. HPB (Oxford). 2010;12:361379.

6. Modlin IM, Lye KD, Kidd M. A 5-decade analysis of 13,715 carcinoid tumors. Cancer. 2003;97:934-959.

7. Modlin IM, Kidd M, Latich I, Zikusoka MN, Shapiro MD. Current status of gastrointestinal carcinoids. Gastroenterology. 2005;128:1717-1751.

8. Bodei L, Mueller-Brand J, Baum RP, et al. The joint IAEA, EANM, and SNMMI practical guidance on peptide receptor radionuclide therapy (PRRNT) in neuroendocrine tumours. Eur J Nucl Med Mol Imaging. 2013;40:800-816.

9. Martini C, Gamper EM, Wintner L, et al. Systematic review reveals lack of quality in reporting health-related quality of life in patients with gastroenteropancreatic neuroendocrine tumours. Health Qual Life Outcomes. 2016;14:127.

10. Pearman TP, Beaumont JL, Cella D, Neary MP, Yao J. Health-related quality of life in patients with neuroendocrine tumors: an investigation of treatment type, disease status, and symptom burden. Support Care Cancer. 2016;24:3695-3703.

11. Larsson G, von Essen L, Sjoden PO. Quality of life in patients with endocrine tumors of the gastrointestinal tract: patient and staff perceptions. Cancer Nurs. 1998;21:411-420.

12. Larsson G, von Essen L, Sjoden PO. Health-related quality of life in patients with endocrine tumours of the gastrointestinal tract. Acta Oncol. 1999;38:481490.

13. Larsson G, Sjoden PO, Oberg K, von Essen L. Importance-satisfaction discrepancies are associated with health-related quality of life in five-year survivors of endocrine gastrointestinal tumours. Ann Oncol. 1999;10:1321-1327.

14. Frojd C, Larsson G, Lampic C, von Essen L. Health related quality of life and psychosocial function among patients with carcinoid tumours: a longitudinal, prospective, and comparative study. Health Qual Life Outcomes. 2007;5:18.

15. Larsson G, Haglund K, Von Essen L. Distress, quality of life and strategies to 'keep a good mood' in patients with carcinoid tumours: patient and staff perceptions. Eur J Cancer Care (Engl). 2003;12:46-57.

16. Haugland T, Vatn MH, Veenstra M, Wahl AK, Natvig GK. Health related quality of life in patients with neuroendocrine tumors compared with the general Norwegian population. Qual Life Res. 2009;18:719-726.

17. Larsson G, Sjoden PO, Oberg K, Eriksson B, von Essen L. Health-related quality of life, anxiety and depression in patients with midgut carcinoid tumours. Acta Oncol. 2001;40:825-831.

18. Hörsch D, Ezziddin S, Haug A, et al. Effectiveness and side-effects of peptide receptor radionuclide therapy for neuroendocrine neoplasms in Germany: a multiinstitutional registry study with prospective follow-up. Eur J Cancer. 2016;58:41-51.

19. Strosberg J, Wolin E, Chasen B, et al. NETTER-1 phase III in patients with midgut neuroendocrine tumors treated with ${ }^{177} \mathrm{Lu}$-dotatate: efficacy, safety, QoL results and subgroup analysis. Ann Oncol. 2016;27:420PD-420PD.

20. Khan S, Krenning EP, van Essen M, Kam BL, Teunissen JJ, Kwekkeboom DJ. Quality of life in 265 patients with gastroenteropancreatic or bronchial neuroendocrine tumors treated with $\left[{ }^{177} \mathrm{Lu}_{-\mathrm{DOTA}}{ }^{0}, \mathrm{Tyr}^{3}\right]$ octreotate. J Nucl Med. 2011;52:1361-1368.

21. Kwekkeboom DJ, Kam BL, van Essen M, et al. Somatostatin-receptor-based imaging and therapy of gastroenteropancreatic neuroendocrine tumors. Endocr Relat Cancer. 2010;17:R53-R73. 
22. van Essen M, Krenning EP, Kam BLR, de Jong M, Valkema R, Kwekkeboom DJ. Peptide-receptor radionuclide therapy for endocrine tumors. Nat Rev Endocrinol. 2009;5:382-393.

23. Kwekkeboom DJ, Mueller-Brand J, Paganelli G, et al. Overview of results of peptide receptor radionuclide therapy with 3 radiolabeled somatostatin analogs. J Nucl Med. 2005;46(suppl):62S-66S

24. Frilling A, Weber F, Saner F, et al. Treatment with ${ }^{90} \mathrm{Y}$ - and ${ }^{177} \mathrm{Lu}-\mathrm{DOTATOC}$ in patients with metastatic neuroendocrine tumors. Surgery. 2006;140:968-976.

25. Teunissen JJ, Kwekkeboom DJ, Krenning EP. Quality of life in patients with gastroenteropancreatic tumors treated with $\left[{ }^{177} \mathrm{Lu}-\mathrm{DOTA}^{0}, \mathrm{Tyr}^{3}\right]$ octreotate. J Clin Oncol. 2004;22:2724-2729.

26. Cwikla JB, Sankowski A, Seklecka N, et al. Efficacy of radionuclide treatment DOTATATE Y-90 in patients with progressive metastatic gastroenteropancreatic neuroendocrine carcinomas (GEP-NETs): a phase II study. Ann Oncol. 2010;21: 787-794.

27. Bodei L, Cremonesi M, Grana CM, et al. Peptide receptor radionuclide therapy with ${ }^{177}$ Lu-DOTATATE: the IEO phase I-II study. Eur J Nucl Med Mol Imaging. 2011;38:2125-2135.

28. Delpassand ES, Samarghandi A, Zamanian S, et al. Peptide receptor radionuclide therapy with ${ }^{177} \mathrm{Lu}$-DOTATATE for patients with somatostatin receptor-expressing neuroendocrine tumors: the first US phase 2 experience. Pancreas. 2014;43: $518-525$.

29. Marinova M, Mucke M, Mahlberg L, et al. Improving quality of life in patients with pancreatic neuroendocrine tumor following peptide receptor radionuclide therapy assessed by EORTC QLQ-C30. Eur J Nucl Med Mol Imaging. 2018;45: $38-46$.

30. Chen J, Ou L, Hollis SJ. A systematic review of the impact of routine collection of patient reported outcome measures on patients, providers and health organisations in an oncologic setting. BMC Health Serv Res. 2013;13:211.

31. Howell D, Molloy S, Wilkinson K, et al. Patient-reported outcomes in routine cancer clinical practice: a scoping review of use, impact on health outcomes, and implementation factors. Ann Oncol. 2015;26:1846-1858.

32. Snyder CF, Aaronson NK, Choucair AK, et al. Implementing patient-reported outcomes assessment in clinical practice: a review of the options and considerations. Qual Life Res. 2012;21:1305-1314.

33. Velikova $\mathrm{G}$, Booth $\mathrm{L}$, Smith $\mathrm{AB}$, et al. Measuring quality of life in routine oncology practice improves communication and patient well-being: a randomized controlled trial. J Clin Oncol. 2004;22:714-724.

34. Unger JM, Hershman DL, Albain KS, et al. Patient income level and cancer clinical trial participation. J Clin Oncol. 2013;31:536-542.

35. Gamper EM, Wintner LM, Rodrigues M, et al. Persistent quality of life impairments in differentiated thyroid cancer patients: results from a monitoring programme. Eur J Nucl Med Mol Imaging. 2015;42:1179-1188.

36. Holzner B, Kemmler G, Cella D, et al. Normative data for functional assessment of cancer therapy: general scale and its use for the interpretation of quality of life scores in cancer survivors. Acta Oncol. 2004;43:153-160.

37. Aaronson NK, Ahmedzai S, Bergman B, et al. The European Organization for Research and Treatment of Cancer QLQ-C30: a quality-of-life instrument for use in international clinical trials in oncology. J Natl Cancer Inst. 1993;85:365-376.

38. Fayers PM, Aaronson NK, Bjordal K, Groenvold M, Curran D, Bottomley A. The EORTC QLQ-C30 Scoring Manual. 3rd ed. Brussels, Belgium: European Organisation for Research and Treatment of Cancer; 2001.
39. Osoba D, Rodrigues G, Myles J, Zee B, Pater J. Interpreting the significance of changes in health-related quality-of-life scores. J Clin Oncol. 1998;16: 139-144.

40. Bergsma H, van Vliet EI, Teunissen JJM, et al. Peptide receptor radionuclide therapy (PRRT) for GEP-NETs. Best Pract Res Clin Gastroenterol. 2012;26: 867-881.

41. Dash A, Chakraborty S, Pillai MR, Knapp FF Jr. Peptide receptor radionuclide therapy: an overview. Cancer Biother Radiopharm. 2015;30:47-71.

42. van der Zwan WA, Bodei L, Mueller-Brand J, de Herder WW, Kvols LK, Kwekkeboom DJ. GEPNETs update: radionuclide therapy in neuroendocrine tumors. Eur J Endocrinol. 2015;172:R1-R8.

43. Gelhorn HL, Kulke MH, O'Dorisio T, et al. Patient-reported symptom experiences in patients with carcinoid syndrome after participation in a study of telotristat etiprate: a qualitative interview approach. Clin Ther. 2016;38:759-768.

44. Beaumont JL, Cella D, Phan AT, Choi S, Liu Z, Yao JC. Comparison of healthrelated quality of life in patients with neuroendocrine tumors with quality of life in the general US population. Pancreas. 2012;41:461-466.

45. Gold CH, Malmberg B, McClearn GE, Pedersen NL, Berg S. Gender and health: a study of older unlike-sex twins. J Gerontol B Psychol Sci Soc Sci. 2002;57(suppl): S168-S176.

46. Silverstein B. Gender difference in the prevalence of clinical depression: the role played by depression associated with somatic symptoms. Am J Psychiatry. 1999; 156:480-482.

47. Kroenke K, Spitzer RL. Gender differences in the reporting of physical and somatoform symptoms. Psychosom Med. 1998;60:150-155.

48. Chan DL, Segelov E, Singh S. Everolimus in the management of metastatic neuroendocrine tumours. Therap Adv Gastroenterol. 2017;10:132-141.

49. Pavel ME, Singh S, Strosberg JR, et al. Health-related quality of life for everolimus versus placebo in patients with advanced, non-functional, well-differentiated gastrointestinal or lung neuroendocrine tumours (RADIANT-4): a multicentre, randomised, double-blind, placebo-controlled, phase 3 trial. Lancet Oncol. 2017; 18:1411-1422.

50. Pavel M, Unger N, Borbath I, et al. Safety and QOL in patients with advanced NET in a phase $3 b$ expanded access study of everolimus. Target Oncol. 2016;11: $667-675$.

51. Raymond E, Dahan L, Raoul JL, et al. Sunitinib malate for the treatment of pancreatic neuroendocrine tumors. N Engl J Med. 2011;364:501-513.

52. Yadegarfar G, Friend L, Jones L, et al. Validation of the EORTC QLQ-GINET21 questionnaire for assessing quality of life of patients with gastrointestinal neuroendocrine tumours. Br J Cancer. 2013;108:301-310.

53. Vinik E, Carlton CA, Silva MP, Vinik AI. Development of the Norfolk quality of life tool for assessing patients with neuroendocrine tumors. Pancreas. 2009;38: e87-e95.

54. Devlin NJ, Appleby J. Getting the Most out of PROMS: Putting Health Outcomes at the Heart of NHS Decision-Making. London, U.K.: The King's Fund; 2010.

55. Aaronson N, Elliott T, Greenhalgh J, et al. User's Guide to Implementing PatientReported Outcomes Assessment in Clinical Practice. Milwaukee, WI: International Society for Quality of Life Research; 2015.

56. Basch E, Snyder C, McNiff K, et al. Patient-reported outcome performance measures in oncology. J Oncol Pract. 2014;10:209-211.

57. Cella D, Hahn EA, Jensen SE, et al. Patient-Reported Outcomes in Performance Measurement. Research Triangle Park, NC: RTI Press; 2015. 